\title{
Global Stability of an Epidemic Model with Incomplete Treatment and Vaccination
}

\author{
Hai-Feng Huo and Li-Xiang Feng \\ Department of Applied Mathematics, Lanzhou University of Technology, Lanzhou, Gansu 730050, China \\ Correspondence should be addressed to Hai-Feng Huo, hfhuo@lut.cn
}

Received 10 December 2011; Revised 30 December 2011; Accepted 30 December 2011

Academic Editor: Binggen Zhang

Copyright (c) 2012 H.-F. Huo and L.-X. Feng. This is an open access article distributed under the Creative Commons Attribution License, which permits unrestricted use, distribution, and reproduction in any medium, provided the original work is properly cited.

An epidemic model with incomplete treatment and vaccination for the newborns and susceptibles is constructed. We establish that the global dynamics are completely determined by the basic reproduction number $R_{0}$. If $R_{0} \leq 1$, then the disease-free equilibrium is globally asymptotically stable. If $R_{0}>1$, the endemic equilibrium is globally asymptotically stable. Some numerical simulations are also given to explain our conclusions.

\section{Introduction}

Epidemiological models describing a directly transmitted viral or bacterial agent in a closed population and consisting of susceptibles $(S)$, infectives $(I)$, and recovers $(R)$ were considered by Kermack and Mckendrick (1927). For some diseases, such as influenza and tuberculosis, on adequate contact with an infectious individual, a susceptible becomes exposed for a while, that is, infected but not yet infectious. Thus it is realistic to introduce a latent compartment (usually denoted by E), leading to an SEIR model [1]. Such type of models has been widely discussed in recent decades [2-8].

Vaccination is important for the elimination of infectious disease. Usually, the vaccination process are different schedules for different disease and vaccines. For some disease, such as hepatitis B virus infection [9], doses should be taken by vaccinees several times and there must be some fixed time intervals between two doses. Considering the time for vaccines to obtain immunity and possibility to be infected before vaccination, Liu et al. [10] studied the vaccination effects via two SVIR models according to continuous vaccination strategy and pulse vaccination strategy (PVS), respectively. Li et al. [11] considered that the vaccine is available for both the susceptibles and the newborns, and the immunity of the vaccinated individuals is temporary and that the efficiency of vaccine is not complete. 
In [12-15], it is assumed that the treated individuals have partial immunity and can be infected through contacts with infectious individuals. Yang et al. [15] incorporated the incomplete treatment into TB epidemic model with treatment, it is assumed that the being treated individuals are kept at an isolated environment, therefore, individuals in treatment compartment are not able to infect others or be infected. Since individual's symptoms of TB may disappear after being treated, but a few of tubercle bacillus may still be left in the body of the treated individual $[16,17]$, then the treated individual may still be a TB carrier and become latent or may enter the infectious compartment for treatment failure [18].

Motivated by these works, in this paper, we consider an SVEIT epidemiological model with varying infectivity. The model assumes that the vaccine is available for both the susceptibles and the newborns and the immunity of the vaccinated individuals is temporary, and that the efficiency of vaccine is not complete. And we also incorporate the incomplete treatment into the epidemic model.

The organization of this paper is as follows. In the next section, the epidemic model with incomplete treatment and vaccination for the newborns and susceptibles is formulated. In Section 3, the basic reproduction number and the existence of equilibria are investigated. The global stability of the disease-free and endemic equilibria are proved in Section 4, and some numerical simulations are given in Section 5. In the last section, we give some brief discussions.

\section{The Model Formulation}

In this section, we introduce an epidemic model with incomplete treatment and vaccination. The total population is partitioned into five compartments: the susceptible compartment $(S)$, the vaccinated compartment $(V)$, the latent compartment $(E)$, the infectious compartment $(I)$, and the treatment compartment $(T)$. The population flow among those compartments is shown in the following diagram (Figure 1).

The schematic diagram leads to the following system of ordinary differential equations:

$$
\begin{gathered}
S^{\prime}=q \mu A-(\mu+p) S-\beta S I+\varepsilon V, \\
V^{\prime}=(1-q) \mu A+p S-\beta \sigma V I-(\mu+\varepsilon) V, \\
E^{\prime}=\beta I(S+\sigma V)-(\mu+\gamma) E+(1-k) \delta T, \\
I^{\prime}=\gamma E-\left(\mu+\alpha_{1}+\xi\right) I+k \delta T, \\
T^{\prime}=\xi I-\left(\mu+\alpha_{2}+\delta\right) T .
\end{gathered}
$$

Here, $\mu A$ is the birth rate of the population; $\mu$ is the natural death rate of the population; $q(0<q<1)$ is the fraction of the unvaccinated newborns, $1-q$ is the fraction of the vaccinated newborns; $p$ is the vaccinating rate coefficient for the susceptible individuals; $\varepsilon$ is the rate coefficient of losing the immunity from the vaccination. $\beta$ and $\sigma \beta(0<\sigma<1)$ are the transmission coefficient of the infection for the susceptible and vaccinated individuals from the infectious individuals, where $0<\sigma<1$ shows that the efficiency of the vaccine is not complete $(100 \%) ; \gamma$ is the rate coefficient of transfer from the latent compartment to the infectious one; $\xi$ is the percapita treatment rate for the infectious individuals; $\delta$ is the rate coefficient at which a treated individual leaves compartment $T ; \alpha_{1}$ and $\alpha_{2}$ are the disease-induced death rate coefficients for individuals in compartments $I$ and $T$, respectively; 


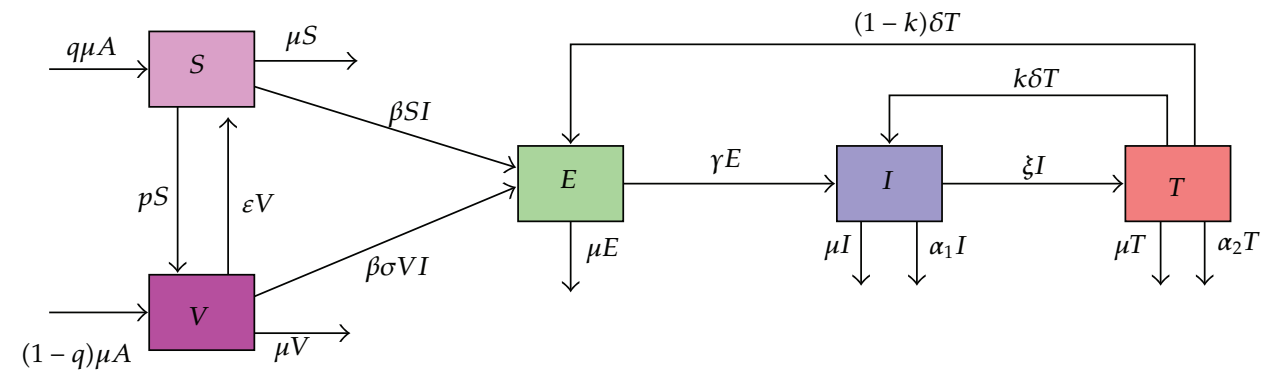

Figure 1: The transfer diagram for the model (2.1).

$k(0 \leq k \leq 1)$ is the fraction of the drug-resistant individuals in the treated individuals. $k$ reflects the failure of treatment, where $k=0$ means that all the treated individuals will become latent, while $k=1$ means that the treatment fails and all the treated individuals will still be infectious.

It is important to show positivity and boundedness for the system (2.1) as they represent populations. Firstly, we present the positivity of the solutions. System (2.1) can be put into the matrix form

$$
X^{\prime}=G(X)
$$

where $X=(S, V, E, I, T)^{T} \in R^{5}$ and $G(X)$ is given by

$$
G(X)=\left(\begin{array}{c}
G_{1}(X) \\
G_{2}(X) \\
G_{3}(X) \\
G_{4}(X) \\
G_{5}(X)
\end{array}\right)=\left(\begin{array}{c}
q \mu A-(\mu+p) S-\beta S I+\varepsilon V \\
(1-q) \mu A+p S-\beta \sigma V I-(\mu+\varepsilon) V \\
\beta I(S+\sigma V)-(\mu+\gamma) E+(1-k) \delta T \\
\gamma E-\left(\mu+\alpha_{1}+\xi\right) I+k \delta T \\
\xi I-\left(\mu+\alpha_{2}+\delta\right) T
\end{array}\right) .
$$

It is easy to check that

$$
\left.G_{i}(X)\right|_{X_{i}(t)=0, X_{t} \in C_{+}} \geq 0, \quad i=1,2,3,4,5 .
$$

Due to Lemma 2 in [19], any solution of (2.1) is such that $X(t) \in R_{+}^{5}$ for all $t \geq 0$.

Summing equations in (2.1) yields

$$
\begin{aligned}
(S+V+E+I+T)^{\prime} & =\mu[A-(S+V+E+I+T)]-\alpha_{1} I-\alpha_{2} T \\
& \leq \mu[A-(S+V+E+I+T)]
\end{aligned}
$$

then it follows that $\lim _{t \rightarrow+\infty} \sup [S(t)+V(t)+E(t)+I(t)+T(t)] \leq A$, so the set

$$
\Omega=\left\{(S, V, E, I, T) \in R_{+}^{5}: S+V+E+I+T \leq A\right\}
$$

is positively invariant for (2.1). Therefore, we will consider the global stability of (2.1) on the set $\Omega$. 


\section{The Basic Reproduction Number and Existence of Equilibria}

The model has a disease-free equilibrium $P_{0}\left(S_{0}, V_{0}, 0,0,0\right)$, where

$$
S_{0}=\frac{A(\varepsilon+q \mu)}{p+\mu+\varepsilon}, \quad V_{0}=\frac{A[p+(1-q) \mu]}{p+\mu+\varepsilon}
$$

In the following, the basic reproduction number of system (2.1) will be obtained by the next generation matrix method formulated in [20].

Let $x=(E, I, T, S, V)^{T}$, then system (2.1) can be written as

$$
\frac{d x}{d t}=\mp(x)-\mho(x)
$$

where

$$
\mathcal{F}(x)=\left(\begin{array}{c}
\beta I(S+\sigma V) \\
0 \\
0 \\
0 \\
0
\end{array}\right), \quad \mho(x)=\left(\begin{array}{c}
(\mu+\gamma) E-(1-k) \delta T \\
\left(\mu+\alpha_{1}+\xi\right) I-\gamma E-k \delta T \\
\left(\mu+\alpha_{2}+\delta\right) T-\xi I \\
\beta S I+(\mu+p) S-q \mu A-\varepsilon V \\
\beta \sigma V I+(\mu+\varepsilon) V-(1-q) \mu A-p S
\end{array}\right)
$$

The Jacobian matrices of $\mathcal{F}(x)$ and $\mho(x)$ at the disease-free equilibrium $P_{0}$ are, respectively,

$$
D \mathcal{F}\left(P_{0}\right)=\left(\begin{array}{cc}
F_{3} \times 3 & 0 \\
0 & 0
\end{array}\right), \quad D \mathcal{U}\left(P_{0}\right)=\left(\begin{array}{ccc}
V_{3} \times 3 & 0 & 0 \\
0 \beta S_{0} 0 & \mu+p & -\varepsilon \\
0 \beta \sigma V_{0} 0 & -p & \mu+\varepsilon
\end{array}\right)
$$

where

$$
F=\left(\begin{array}{ccc}
0 & \beta\left(S_{0}+\sigma V_{0}\right) & 0 \\
0 & 0 & 0 \\
0 & 0 & 0
\end{array}\right), \quad V=\left(\begin{array}{ccc}
\mu+\gamma & 0 & -(1-k) \delta \\
-\gamma & \mu+\alpha_{1}+\xi & -k \delta \\
0 & -\xi & \mu+\alpha_{2}+\delta
\end{array}\right)
$$

The model reproduction number, denoted by $R_{0}$ is thus given by

$$
R_{0}=\rho\left(F V^{-1}\right)=\frac{\beta \gamma\left(\mu+\alpha_{2}+\delta\right)\left(S_{0}+\sigma V_{0}\right)}{(\mu+\gamma)\left(\mu+\alpha_{1}+\xi\right)\left(\mu+\alpha_{2}+\delta\right)-\delta \xi[(1-k) \gamma+(\mu+\gamma) k]}
$$


The endemic equilibrium $P^{*}\left(S^{*}, V^{*}, E^{*}, I^{*}, T^{*}\right)$ of system (2.1) is determined by equations

$$
\begin{gathered}
q \mu A-(\mu+p) S-\beta S I+\varepsilon V=0, \\
(1-q) \mu A+p S-\beta \sigma V I-(\mu+\varepsilon) V=0, \\
\beta I(S+\sigma V)-(\mu+\gamma) E+(1-k) \delta T=0, \\
\gamma E-\left(\mu+\alpha_{1}+\xi\right) I+k \delta T=0, \\
\xi I-\left(\mu+\alpha_{2}+\delta\right) T=0 .
\end{gathered}
$$

The first two equations in (3.7) lead to

$$
S=\frac{\mu A[q(\mu+\varepsilon+\beta \sigma I)+\varepsilon(1-q)]}{(p+\mu+\beta I)(\mu+\varepsilon+\beta \sigma I)-p \varepsilon}, \quad V=\frac{\mu A[(1-q)(p+\mu+\beta I)+p q]}{(p+\mu+\beta I)(\mu+\varepsilon+\beta \sigma I)-p \varepsilon} .
$$

From the last equation in (3.7), we have

$$
T=\frac{\xi}{\mu+\alpha_{2}+\delta} I
$$

Substituting (3.9) into the fourth equation in (3.7) gives

$$
E=\frac{\left(\mu+\alpha_{1}+\xi\right)\left(\mu+\alpha_{2}+\delta\right)-k \delta \xi}{\left(\mu+\alpha_{2}+\delta\right) \gamma} I
$$

For $I \neq 0$, substituting (3.9), (3.10) into the third equation in (3.7) gives

$$
S+\sigma V=\frac{1}{\beta} \frac{(\mu+\gamma)\left[\left(\mu+\alpha_{1}+\xi\right)\left(\mu+\alpha_{2}+\delta\right)-k \delta \xi\right]-(1-k) \delta \xi \gamma}{\left(\mu+\alpha_{2}+\delta\right) \gamma}
$$

Substituting (3.8) into (3.11) yields

$$
\begin{aligned}
H(I):= & \frac{[q(\mu+\varepsilon+\beta \sigma I)+\varepsilon(1-q)]+\sigma[(1-q)(p+\mu+\beta I)+p q]}{(p+\mu+\beta I)(\mu+\varepsilon+\beta \sigma I)-p \varepsilon} \\
& -\frac{(\mu+\gamma)\left[\left(\mu+\alpha_{1}+\xi\right)\left(\mu+\alpha_{2}+\delta\right)-k \delta \xi\right]-(1-k) \delta \xi \gamma}{\beta \gamma\left(\mu+\alpha_{2}+\delta\right) \mu A} \\
= & \frac{\beta \sigma I+(\varepsilon+q \mu)+\sigma[p+(1-q) \mu]}{(p+\mu+\beta I)(\mu+\varepsilon+\beta \sigma I)-p \varepsilon} \\
& -\frac{(\mu+\gamma)\left[\left(\mu+\alpha_{1}+\xi\right)\left(\mu+\alpha_{2}+\delta\right)-k \delta \xi\right]-(1-k) \delta \xi \gamma}{\beta \gamma\left(\mu+\alpha_{2}+\delta\right) \mu A} \\
= & 0 .
\end{aligned}
$$


Direct calculation shows

$$
\frac{H^{\prime}(I)}{\beta}=-\frac{(\beta \sigma I)^{2}+2 \beta \sigma I(\varepsilon+q \mu)+\sigma[p+(1-q) \mu]+\sigma_{0}}{[(p+\mu+\beta I)(\mu+\varepsilon+\beta \sigma I)-p \varepsilon]^{2}}<0
$$

where

$$
\sigma_{0}=(p+\mu)[p+(1-q) \mu] \sigma^{2}+\sigma[p(\varepsilon+q \mu)+\varepsilon(p+(1-q) \mu)]+(\mu+\varepsilon)(\varepsilon+q \mu)
$$

then function $H(I)$ is decreasing for $I>0$. Since $(p+\mu+\beta I)(\mu+\varepsilon+\beta \sigma I)-p \varepsilon>\beta I(\beta \sigma I+\varepsilon+\sigma p+\mu)$, and it follows from $\sigma \leq 1$ that $\beta \sigma I+(\varepsilon+q \mu)+\sigma[p+(1-q) \mu]<\beta \sigma I+\varepsilon+\sigma p+\mu$, then

$$
H(I)<\frac{1}{\beta I}-\frac{(\mu+\gamma)\left[\left(\mu+\alpha_{1}+\xi\right)\left(\mu+\alpha_{2}+\delta\right)-k \delta \xi\right]-(1-k) \delta \xi \gamma}{\beta \gamma\left(\mu+\alpha_{2}+\delta\right) \mu A}
$$

Thus,

$$
\begin{aligned}
H(0) & =\frac{(\varepsilon+q \mu)+\sigma[p+(1-q) \mu]}{\mu(p+\mu+\varepsilon)}-\frac{(\mu+\gamma)\left[\left(\mu+\alpha_{1}+\xi\right)\left(\mu+\alpha_{2}+\delta\right)-k \delta \xi\right]-(1-k) \delta \xi \gamma}{\beta \gamma\left(\mu+\alpha_{2}+\delta\right) \mu A} \\
& =\frac{(\mu+\gamma)\left[\left(\mu+\alpha_{1}+\xi\right)\left(\mu+\alpha_{2}+\delta\right)-k \delta \xi\right]-(1-k) \delta \xi \gamma}{\beta \gamma\left(\mu+\alpha_{2}+\delta\right) \mu A}\left(R_{0}-1\right), \\
H(A) & <\frac{1}{\beta A}-\frac{(\mu+\gamma)\left[\left(\mu+\alpha_{1}+\xi\right)\left(\mu+\alpha_{2}+\delta\right)-k \delta \xi\right]-(1-k) \delta \xi \gamma}{\beta \gamma\left(\mu+\alpha_{2}+\delta\right) \mu A} \\
& =\frac{\left(\mu+\alpha_{2}+\delta\right)\left[\gamma \mu-(\mu+\gamma)\left(\mu+\alpha_{1}+\xi\right)\right]+\delta \xi(\gamma+\mu k)}{\beta A \gamma \mu\left(\mu+\alpha_{2}+\delta\right)} \\
& =\frac{-\left(\mu+\alpha_{2}+\delta\right)\left[\mu\left(\mu+\alpha_{1}+\xi\right)+\gamma\left(\alpha_{1}+\xi\right)\right]+\delta \xi(\gamma+\mu k)}{\beta A \gamma \mu\left(\mu+\alpha_{2}+\delta\right)} \\
& =-\frac{\left(\mu+\alpha_{2}+\delta\right)\left[\mu\left(\mu+\alpha_{1}\right)+\gamma \alpha_{1}\right]+\xi(\mu+\gamma)\left(\mu+\alpha_{2}\right)+\mu \delta \xi(1-k)}{\beta A \gamma \mu\left(\mu+\alpha_{2}+\delta\right)} . \\
& <0 .
\end{aligned}
$$

Therefore, by the monotonicity of function $H(I)$, for (3.12) there exists a unique positive root in the interval $(0, A)$ when $R_{0}>1$; there is no positive root in the interval $(0, A)$ when $R_{0} \leq 1$. We summarize this result in Theorem 3.1. 
Theorem 3.1. For system (2.1), there is always the disease-free equilibrium $P_{0}\left(S_{0}, V_{0}, 0,0,0\right)$. When $R_{0}>1$, besides the disease-free equilibrium $P_{0}$, system (2.1) also has a unique endemic equilibrium $P^{*}\left(S^{*}, V^{*}, E^{*}, I^{*}, T^{*}\right)$, where

$$
\begin{gathered}
S^{*}=\frac{\mu A\left[q\left(\mu+\varepsilon+\beta \sigma I^{*}\right)+\varepsilon(1-q)\right]}{\left(p+\mu+\beta I^{*}\right)\left(\mu+\varepsilon+\beta \sigma I^{*}\right)-p \varepsilon}, \\
V^{*}=\frac{\mu A\left[(1-q)\left(p+\mu+\beta I^{*}\right)+p q\right]}{\left(p+\mu+\beta I^{*}\right)\left(\mu+\varepsilon+\beta \sigma I^{*}\right)-p \varepsilon}, \\
E^{*}=\frac{\left(\mu+\alpha_{1}+\xi\right)\left(\mu+\alpha_{2}+\delta\right)-k \delta \xi}{\left(\mu+\alpha_{2}+\delta\right) \gamma} I^{*}, \\
T^{*}=\frac{\xi}{\mu+\alpha_{2}+\delta} I^{*},
\end{gathered}
$$

and $I^{*}$ is the unique positive root of equation $H(I)=0$.

\section{Global Stability of Equilibria}

Theorem 4.1. For system (2.1), the disease-free equilibrium $P_{0}$ is globally stable if $R_{0} \leq 1$; the endemic equilibrium $P^{*}$ is globally stable if $R_{0}>1$.

\subsection{The Proof Global Stability of the Disease-Free Equilibrium}

For the disease-free equilibrium $P_{0}\left(S_{0}, V_{0}, 0,0,0\right), S_{0}$ and $V_{0}$ satisfy equations

$$
\begin{gathered}
q \mu A-(\mu+p) S-\beta S I+\varepsilon V=0, \\
(1-q) \mu A+p S-\beta \sigma V I-(\mu+\varepsilon) V=0,
\end{gathered}
$$

then (2.1) can be rewritten as follows:

$$
\begin{gathered}
S^{\prime}=S\left[q \mu A\left(\frac{1}{S}-\frac{1}{S_{0}}\right)+\varepsilon\left(\frac{V}{S}-\frac{V_{0}}{S_{0}}\right)-\beta I\right], \\
V^{\prime}=V\left[(1-q) \mu A\left(\frac{1}{V}-\frac{1}{V_{0}}\right)+p\left(\frac{V}{S}-\frac{V_{0}}{S_{0}}\right)-\beta \sigma I\right], \\
E^{\prime}=\beta I\left[\left(S_{0}+\sigma V_{0}\right)+\left(S-S_{0}\right)+\sigma\left(V-V_{0}\right)\right]-(\mu+\gamma) E+(1-k) \delta T, \\
I^{\prime}=\gamma E-\left(\mu+\alpha_{1}+\xi\right) I+k \delta T, \\
T^{\prime}=\xi I-\left(\mu+\alpha_{2}+\delta\right) T .
\end{gathered}
$$

Define the Lyapunov function

$$
V_{1}=\left(S-S_{0}-S_{0} \ln \frac{S}{S_{0}}\right)+\left(V-V_{0}-V_{0} \ln \frac{V}{V_{0}}\right)+E+\frac{\mu+\gamma}{\gamma} I+\frac{\delta[(\mu+\gamma) k+\gamma(1-k)]}{\gamma\left(\mu+\alpha_{2}+\delta\right)} T
$$


The derivative of $V_{1}$ is given by

$$
\begin{aligned}
V_{1}^{\prime}= & \left(S-S_{0}\right)\left[q \mu A\left(\frac{1}{S}-\frac{1}{S_{0}}\right)+\varepsilon\left(\frac{V}{S}-\frac{V_{0}}{S_{0}}\right)-\beta I\right] \\
& +\left(V-V_{0}\right)\left[(1-q) \mu A\left(\frac{1}{V}-\frac{1}{V_{0}}\right)+p\left(\frac{V}{S}-\frac{V_{0}}{S_{0}}\right)-\beta \sigma I\right] \\
& +\beta I\left[\left(S_{0}+\sigma V_{0}\right)+\left(S-S_{0}\right)+\sigma\left(V-V_{0}\right)\right]-(\mu+\gamma) E+(1-k) \delta T \\
& +\frac{\mu+\gamma}{\gamma}\left[\gamma E-\left(\mu+\alpha_{1}+\xi\right) I+k \delta T\right] \\
& +\frac{\delta[(\mu+\gamma) k+\gamma(1-k)]}{\gamma\left(\mu+\alpha_{2}+\delta\right)}\left[\xi I-\left(\mu+\alpha_{2}+\delta\right) T\right] \\
= & \frac{(\mu+\gamma)\left[\left(\mu+\alpha_{1}+\xi\right)\left(\mu+\alpha_{2}+\delta\right)-\delta \xi[(1-k) \gamma+(\mu+\gamma) k]\right.}{\gamma\left(\mu+\alpha_{2}+\delta\right)}\left(R_{0}-1\right) I+F(S, I),
\end{aligned}
$$

where

$$
\begin{aligned}
F(S, I)= & \mu q A\left(S-S_{0}\right)\left(\frac{1}{S}-\frac{1}{S_{0}}\right)+(1-q) \mu A\left(V-V_{0}\right)\left(\frac{1}{V}-\frac{1}{V_{0}}\right) \\
& +\varepsilon\left(S-S_{0}\right)\left(\frac{V}{S}-\frac{V_{0}}{S_{0}}\right)+p\left(V-V_{0}\right)\left(\frac{S}{V}-\frac{S_{0}}{V_{0}}\right) .
\end{aligned}
$$

Denote $x=S / S_{0}, y=V / V_{0}$; then

$$
\begin{aligned}
F(S, I)= & \mu q A(x-1)\left(\frac{1}{x}-1\right)+(1-q) \mu A(y-1)\left(\frac{1}{y}-1\right) \\
& +\varepsilon V_{0}(x-1)\left(\frac{y}{x}-1\right)+p S_{0}(y-1)\left(\frac{x}{y}-1\right)=: \bar{F}(x, y) .
\end{aligned}
$$

Applying (4.1) to function $\bar{F}(x, y)$ yields

$$
\begin{aligned}
\bar{F}(x, y)= & \left(2 \mu q A+2 \mu(1-q) A+\varepsilon V_{0}+p S_{0}\right)-\mu S_{0} x-\mu q A \frac{1}{x}-\mu V_{0} y \\
& -\mu(1-q) A \frac{1}{y}-\varepsilon V_{0} \frac{y}{x}-p S_{0} \frac{x}{y}
\end{aligned}
$$

By [11], we have $\bar{F}(x, y) \leq 0$ for $x, y>0$ and $\bar{F}(x, y)=0$ if and only if $x=y=1$. Since $(\mu+$ $\gamma)\left(\mu+\alpha_{1}+\xi\right)\left(\mu+\alpha_{2}+\delta\right)>\delta \xi[(1-k) \gamma+(\mu+\gamma) k]$ and $R_{0} \leq 1$, then $V_{1}^{\prime} \leq 0$. It follows from LaSalle invariance principle [21] that the disease-free equilibrium $P_{0}$ is globally asymptotically stable when $R_{0} \leq 1$. 


\subsection{The Proof Global Stability of the Endemic Equilibrium}

For the endemic equilibrium $P^{*}\left(S^{*}, V^{*}, E^{*}, I^{*}, T^{*}\right), S^{*}, V^{*}, E^{*}, I^{*}$, and $T^{*}$ satisfy equations

$$
\begin{gathered}
q \mu A-(\mu+p) S-\beta S I+\varepsilon V=0, \\
(1-q) \mu A+p S-\beta \sigma V I-(\mu+\varepsilon) V=0, \\
\beta I(S+\sigma V)-(\mu+\gamma) E+(1-k) \delta T=0, \\
\gamma E-\left(\mu+\alpha_{1}+\xi\right) I+k \delta T=0, \\
\xi I-\left(\mu+\alpha_{2}+\delta\right) T=0
\end{gathered}
$$

which will be used many times in the following inference.

By applying (4.8) and denoting

$$
\frac{S}{S^{*}}=x, \quad \frac{V}{V^{*}}=y, \quad \frac{E}{E^{*}}=z, \quad \frac{I}{I^{*}}=u, \quad \frac{T}{T^{*}}=w,
$$

we have

$$
\begin{gathered}
x^{\prime}=x\left[\frac{q \mu A}{S^{*}}\left(\frac{1}{x}-1\right)-\beta I^{*}(u-1)+\frac{\varepsilon V^{*}}{S^{*}}\left(\frac{y}{x}-1\right)\right], \\
y^{\prime}=y\left[\frac{(1-q) \mu A}{V^{*}}\left(\frac{1}{y}-1\right)+\frac{p S^{*}}{V^{*}}\left(\frac{x}{y}-1\right)-\beta \sigma I^{*}(u-1)\right], \\
z^{\prime}=z \frac{\beta I^{*}}{E^{*}}\left[S^{*}\left(\frac{x u}{z}-1\right)+\sigma V^{*}\left(\frac{y u}{z}-1\right)\right]+z(1-k) \delta \frac{T^{*}}{E^{*}}\left(\frac{w}{z}-1\right), \\
u^{\prime}=u\left[\gamma \frac{E^{*}}{I^{*}}\left(\frac{z}{u}-1\right)+k \delta \frac{T^{*}}{I^{*}}\left(\frac{w}{u}-1\right)\right], \\
w^{\prime}=w \frac{\xi I^{*}}{T^{*}}\left(\frac{u}{w}-1\right) .
\end{gathered}
$$

Define the Lyapunov function

$$
\begin{aligned}
V_{2}= & S^{*}(x-1-\ln x)+V^{*}(y-1-\ln y)+E^{*}(z-1-\ln z)+\frac{\mu+\gamma}{\gamma} I^{*}(u-1-\ln u) \\
& +\frac{\delta[(\mu+\gamma) k+\gamma(1-k)]}{\gamma\left(\mu+\alpha_{2}+\delta\right)} T^{*}(w-1-\ln w) .
\end{aligned}
$$

The derivative of $V_{2}$ is given by

$$
\begin{aligned}
V_{2}^{\prime}= & S^{*} \frac{x-1}{x} x^{\prime}+V^{*} \frac{y-1}{y} y^{\prime}+E^{*} \frac{z-1}{z} z^{\prime}+\frac{\mu+\gamma}{\gamma} I^{*} \frac{u-1}{u} u^{\prime}+\frac{\delta[(\mu+\gamma) k+\gamma(1-k)]}{\gamma\left(\mu+\alpha_{2}+\delta\right)} T^{*} \frac{w-1}{w} w^{\prime} \\
= & (x-1)\left[q \mu A\left(\frac{1}{x}-1\right)-\beta S^{*} I^{*}(u-1)+\varepsilon V^{*}\left(\frac{y}{x}-1\right)\right] \\
& +(y-1)\left[(1-q) \mu A\left(\frac{1}{y}-1\right)+p S^{*}\left(\frac{x}{y}-1\right)-\beta \sigma V^{*} I^{*}(u-1)\right]
\end{aligned}
$$




$$
\begin{aligned}
& +\beta I^{*}(z-1)\left[S^{*}\left(\frac{x u}{z}-1\right)+\sigma V^{*}\left(\frac{y u}{z}-1\right)\right]+(z-1)(1-k) \delta T^{*}\left(\frac{w}{z}-1\right) \\
& +\frac{\mu+\gamma}{\gamma}(u-1)\left[\gamma E^{*}\left(\frac{z}{u}-1\right)+k \delta T^{*}\left(\frac{w}{u}-1\right)\right]+\frac{\delta[(\mu+\gamma) k+\gamma(1-k)]}{\gamma\left(\mu+\alpha_{2}+\delta\right)} \xi I^{*}(w-1)\left(\frac{u}{w}-1\right) \\
& =q \mu A(x-1)\left(\frac{1}{x}-1\right)-\beta S^{*} I^{*}(x-1)(u-1)+\varepsilon V^{*}(x-1)\left(\frac{y}{x}-1\right) \\
& +(1-q) \mu A(y-1)\left(\frac{1}{y}-1\right)+p S^{*}(y-1)\left(\frac{x}{y}-1\right)-\beta \sigma V^{*} I^{*}(y-1)(u-1) \\
& +\beta S^{*} I^{*}(z-1)\left(\frac{x u}{z}-1\right)+\beta \sigma V^{*} I^{*}(z-1)\left(\frac{y u}{z}-1\right)+(1-k) \delta T^{*}(z-1)\left(\frac{w}{z}-1\right) \\
& +(\mu+\gamma) E^{*}(u-1)\left(\frac{z}{u}-1\right)+\frac{\mu+\gamma}{\gamma} k \delta T^{*}(u-1)\left(\frac{w}{u}-1\right)+\frac{[(\mu+\gamma) k+\gamma(1-k)] \delta T^{*}}{\gamma} \\
& \times(w-1)\left(\frac{u}{w}-1\right) \\
& =q \mu A\left(2-x-\frac{1}{x}\right)+(1-q) \mu A\left(2-y-\frac{1}{y}\right)-\beta S^{*} I^{*}(x u-x-u+1)+\varepsilon V^{*}\left(y-x-\frac{y}{x}+1\right) \\
& +p S^{*}\left(x-y-\frac{x}{y}+1\right)-\beta \sigma V^{*} I^{*}(y u-y-u+1)+\beta S^{*} I^{*}\left(x u-z-\frac{x u}{z}+1\right) \\
& +\beta \sigma V^{*} I^{*}\left(y u-z-\frac{y u}{z}+1\right)+(1-k) \delta T^{*}\left(w-z-\frac{w}{z}+1\right)+(\mu+\gamma) E^{*}\left(z-u-\frac{z}{u}+1\right) \\
& +\frac{\mu+\gamma}{\gamma} k \delta T^{*}\left(w-u-\frac{w}{u}+1\right)+\frac{[(\mu+\gamma) k+\gamma(1-k)] \delta T^{*}}{r}\left(u-w-\frac{u}{w}+1\right) \\
& =2 q \mu A+2(1-q) \mu A+\varepsilon V^{*}+p S^{*}+(1-k) \delta T^{*}+(\mu+\gamma) E^{*}+\frac{\mu+\gamma}{\gamma} k \delta T^{*} \\
& +\frac{(\mu+\gamma) k+\gamma(1-k)}{\gamma} \delta T^{*}-\mu S^{*} x-q \mu A \frac{1}{x}-\mu V^{*} y-(1-q) \mu A \frac{1}{y} \\
& +z\left[-\beta S^{*} I^{*}-\beta \sigma V^{*} I^{*}-(1-k) \delta T^{*}+(\mu+\gamma) E^{*}\right] \\
& +u\left[\beta S^{*} I^{*}+\beta \sigma V^{*} I^{*}-(\mu+\gamma) E^{*}-\frac{\mu+\gamma}{\gamma} k \delta T^{*}+\frac{(\mu+\gamma) k+\gamma(1-k)}{\gamma} \delta T^{*}\right] \\
& +w\left[(1-k) \delta T^{*}+\frac{\mu+\gamma}{\gamma} k \delta T^{*}-\frac{(\mu+\gamma) k+\gamma(1-k)}{\gamma} \delta T^{*}\right]-\varepsilon V^{*} \frac{y}{x}-p S^{*} \frac{x}{y}-\beta S^{*} I^{*} \frac{x u}{z} \\
& -\beta \sigma V^{*} I^{*} \frac{y u}{z}-(\mu+\gamma) E^{*} \frac{z}{u}-(1-k) \delta T^{*} \frac{w}{z}-\frac{\mu+\gamma}{\gamma} k \delta T^{*} \frac{w}{u}-\frac{(\mu+\gamma) k+\gamma(1-k)}{\gamma} \delta T^{*} \frac{u}{w} \\
& =\left[2 q \mu A+2(1-q) \mu A+\varepsilon V^{*}+p S^{*}+\beta I^{*}\left(S^{*}+\sigma V^{*}\right)+3(1-k) \delta T^{*}+2 \frac{\mu+\gamma}{\gamma} k \delta T^{*}\right]-\mu S^{*} x \\
& -q \mu A \frac{1}{x}-\mu V^{*} y-(1-q) \mu A \frac{1}{y}-\varepsilon V^{*} \frac{y}{x}-p S^{*} \frac{x}{y}-\beta S^{*} I^{*} \frac{x u}{z}-\beta \sigma V^{*} I^{*} \frac{y u}{z}-\beta I^{*}\left(S^{*}+\sigma V^{*}\right) \frac{z}{u} \\
& -(1-k) \delta T^{*} \frac{z}{u}-(1-k) \delta T^{*} \frac{w}{z}-\frac{\mu+\gamma}{\gamma} k \delta T^{*} \frac{w}{u}-\frac{[(\mu+\gamma) k+\gamma(1-k)]}{\gamma} \delta T^{*} \frac{u}{w}
\end{aligned}
$$


Discrete Dynamics in Nature and Society

$$
\begin{aligned}
= & {\left[2 q \mu A+2(1-q) \mu A+\varepsilon V^{*}+p S^{*}+\beta I^{*}\left(S^{*}+\sigma V^{*}\right)\right]-\mu S^{*} x-q \mu A \frac{1}{x} } \\
& -\mu V^{*} y-(1-q) \mu A \frac{1}{y}-\varepsilon V^{*} \frac{y}{x}-p S^{*} \frac{x}{y}-\beta S^{*} I^{*} \frac{x u}{z}-\beta \sigma V^{*} I^{*} \frac{y u}{z}-\beta I^{*}\left(S^{*}+\sigma V^{*}\right) \frac{z}{u} \\
& +(1-k) \delta T^{*}\left(3-\frac{z}{u}-\frac{w}{z}-\frac{u}{w}\right)+\frac{\mu+\gamma}{\gamma} k \delta T^{*}\left(2-\frac{w}{u}-\frac{u}{w}\right) \\
= & \bar{F}(x, y, z, u)+(1-k) \delta T^{*}\left(3-\frac{z}{u}-\frac{w}{z}-\frac{u}{w}\right)+\frac{\mu+\gamma}{\gamma} k \delta T^{*}\left(2-\frac{w}{u}-\frac{u}{w}\right) .
\end{aligned}
$$

Following [11], $\beta I^{*}\left(S^{*}+\sigma V^{*}\right)=(\mu+\gamma) E^{*}$ and

$$
\begin{aligned}
F(x, y, z, u)= & {\left[2 q \mu A+2(1-q) \mu A+\varepsilon V^{*}+p S^{*}+(\mu+\gamma) E^{*}\right]-\mu S^{*} x-q \mu A \frac{1}{x}-\mu V^{*} y } \\
& -(1-q) \mu A \frac{1}{y}-\varepsilon V^{*} \frac{y}{x}-p S^{*} \frac{x}{y}-\beta S^{*} I^{*} \frac{x u}{z}-\beta \sigma V^{*} I^{*} \frac{y u}{z}-(\mu+\gamma) E^{*} \frac{z}{u} \\
\leq & 0,
\end{aligned}
$$

we have $\bar{F}(x, y, z, u) \leq 0$ for $x, y, z, u>0$ and $\bar{F}(x, y, z, u)=0$ if and only if $x=y=1$ and $z=u$. Therefore, $V_{2}^{\prime} \leq 0$ for $x, y, z, u, w>0$ and $V_{2}^{\prime}=0$ if and only if $x=y=1, z=u=w$, and the maximum invariant set of system (2.1) on the set $\left\{(x, y, z, u, w): V_{2}^{\prime}=0\right\}$ is the singleton $(1,1,1,1,1)$. Thus, for system $(2.1)$, the endemic equilibrium $P^{*}$ is globally asymptotically stable if $R_{0}>1$ by LaSalle invariance principle [21].

\section{Numerical Simulation}

In this section, some numerical results of system (2.1) are presented for supporting the analytic results obtained above. All of the parameter values are estimated. The model parameters are taken as: $A=55.496, \mu=0.0143, q=0.6, \beta=0.05, \sigma=0.2, p=0.05, \varepsilon=0.03$, $\gamma=0.00368, \delta=1.5, \alpha_{1}=0.3, \alpha_{2}=0.05$. First, we choose $\xi=0.9, k=0.3$, numerical simulation gives $R_{0}=0.8809<1$, then the disease-free equilibrium $P_{0}$ is globally asymptotically stable (Figure 2). Second, we choose $\xi=0.8, k=0.36$, numerical simulation gives $R_{0}=1$, then the disease-free equilibrium $P_{0}$ is globally asymptotically stable (Figure 3 ). At last, we choose $\xi=0.6, k=0.5$, numerical simulation gives $R_{0}=1.2938>1$, then the endemic equilibrium $P^{*}$ is globally asymptotically stable (Figure 4 ).

\section{Discussion}

We have formulated an epidemic model with incomplete treatment and vaccination and investigated their dynamical behaviors. By means of the next-generation matrix, we obtained their basic reproduction number, $R_{0}$, which play a crucial role. By constructing Lyapunov function, we proved the global stability of their equilibria: when the basic reproduction number is less than or equal to one, all solutions converge to the disease-free equilibrium, that is, the disease dies out eventually; when the basic reproduction number exceeds one, 


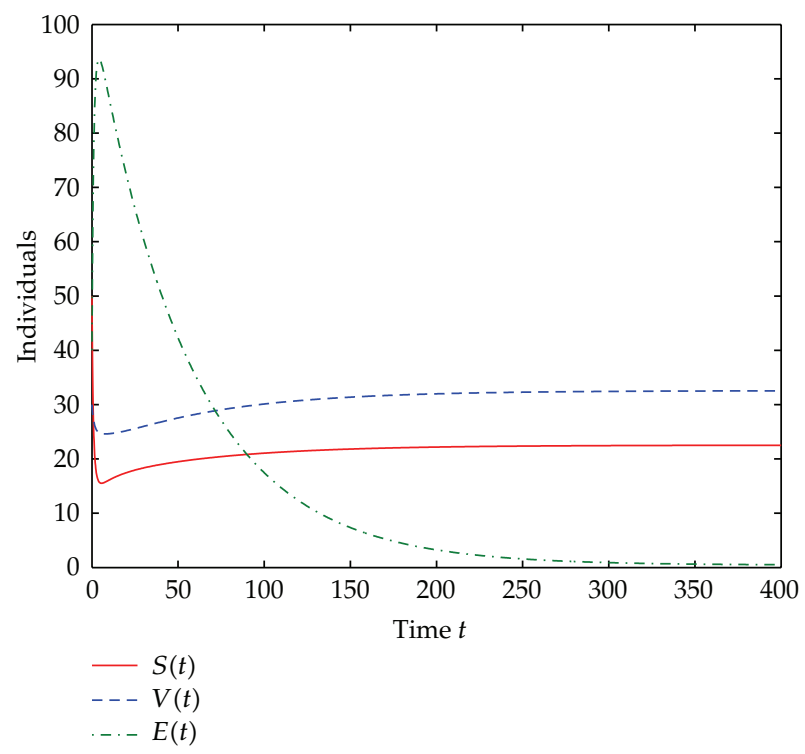

Figure 2: When $R_{0}<1$, the disease-free equilibrium $P_{0}$ is globally asymptotically stable.

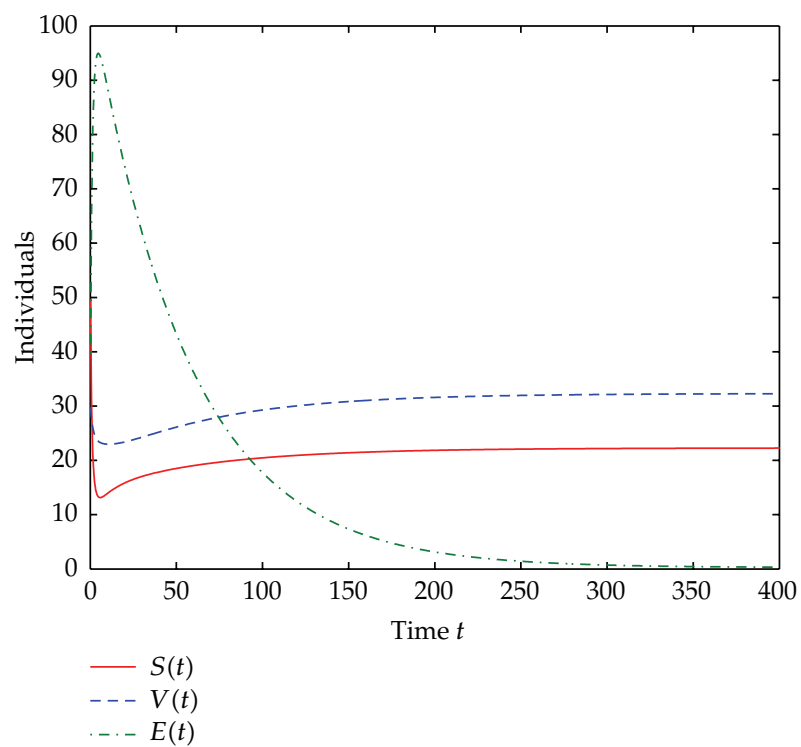

Figure 3: When $R_{0}=1$, the disease-free equilibrium $P_{0}$ is globally asymptotically stable.

the unique endemic equilibrium is globally stable, that is, the disease will persist in the population and the number of infected individuals tends to a positive constant.

For system (2.1), $k$ reflects the failure of treatment. Direct calculation shows that $\partial R_{0} / \partial k>0$, then decreasing the treatment failure coefficient is helpful to reduce epidemic infection. The realization of decreasing $k$ mainly depends on decreasing the appearance of drug resistance. On the other hand, $\partial R_{0} / \partial \xi<0$ implies that increasing $\xi$ has positive effect on epidemic control, since increasing $\xi$ is to shorten the period of the infectious compartment in the nonisolated environment, that is, to start treating as soon as possible. This measure is 


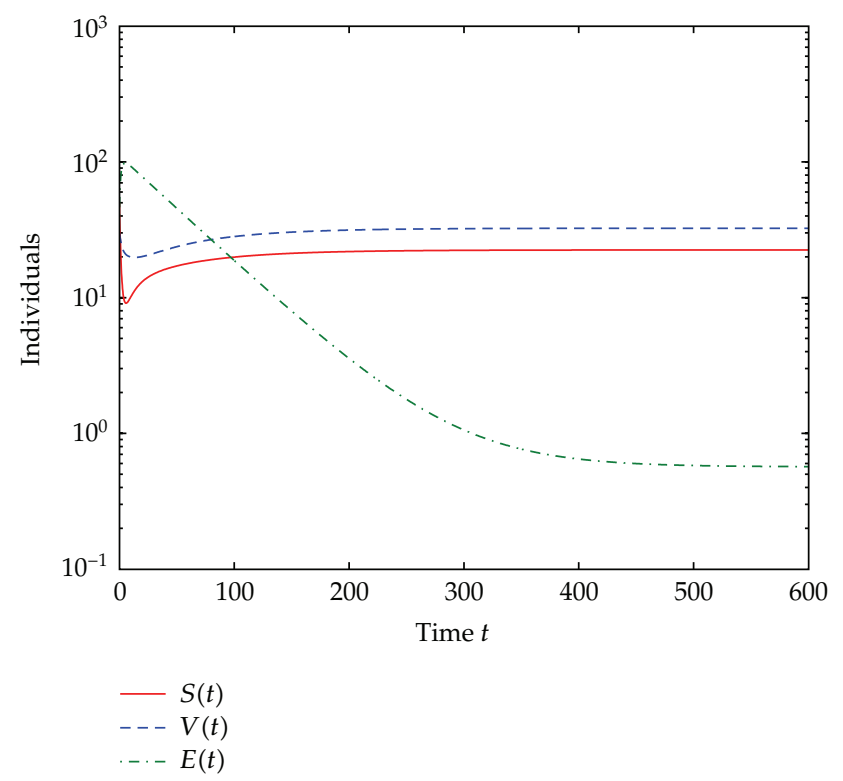

Figure 4: When $R_{0}>1$, the endemic equilibrium $P^{*}$ is globally asymptotically stable.

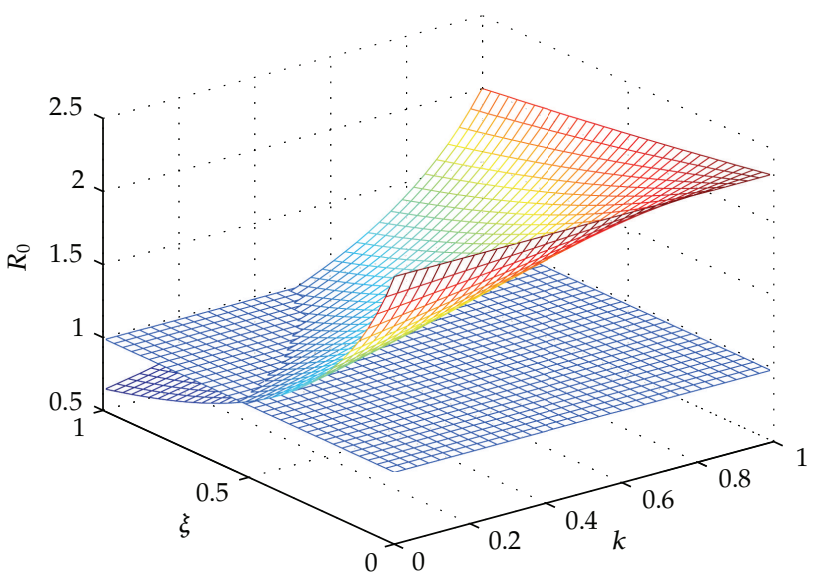

Figure 5: The relationship among $R_{0}, k$ and $\xi$.

effective to control the spread of the epidemic. Figure 5 shows the relation among the basic reproduction number $R_{0}$, the treatment failure coefficient $k$, and the per-capita treatment rate $\xi$.

\section{Acknowledgments}

This work was partially supported by the NNSF of China (10961018), the NSF of Gansu Province of China (1107RJZA088), the NSF for Distinguished Young Scholars of Gansu Province of China (1111RJDA003), the Special Fund for the Basic Requirements in the Research of University of Gansu Province of China, and the Development Program for HongLiu Distinguished Young Scholars in Lanzhou University of Technology. 


\section{References}

[1] J. L. Wang, G. Huang, Y. Takeuchi, and S. Q. Liu, "Sveir epidemiological model with varying infectivity and distributed delays," Mathematical Biosciences and Engineering, vol. 8, no. 3, pp. 875-888, 2011.

[2] J.-J. Wang, J.-Z. Zhang, and Z. Jin, "Analysis of an SIR model with bilinear incidence rate," Nonlinear Analysis: Real World Applications, vol. 11, no. 4, pp. 2390-2402, 2010.

[3] X. Tian and R. Xu, "Stability analysis of a delayed SIR epidemic model with stage structure and nonlinear incidence," Discrete Dynamics in Nature and Society, vol. 2009, Article ID 979217, 17 pages, 2009.

[4] S. Yuan and B. Li, "Global dynamics of an epidemic model with a ratio-dependent nonlinear incidence rate," Discrete Dynamics in Nature and Society, vol. 2009, Article ID 609306, 13 pages, 2009.

[5] J. Zhang, Z. Jin, Y. Xue, and Y. Li, "The dynamics of the pulse birth in an SIR epidemic model with standard incidence," Discrete Dynamics in Nature and Society, vol. 2009, Article ID 490437, 18 pages, 2009.

[6] H.-F. Huo, S.-J. Dang, and Y.-N. Li, "Stability of a two-strain tuberculosis model with general contact rate," Abstract and Applied Analysis, vol. 2010, Article ID 293747, 31 pages, 2010.

[7] M. Y. Li, J. R. Graef, L. Wang, and J. Karsai, “Global dynamics of a SEIR model with varying total population size," Mathematical Biosciences, vol. 160, no. 2, pp. 191-213, 1999.

[8] M. Y. Li and J. S. Muldowney, "Global stability for the SEIR model in epidemiology," Mathematical Biosciences, vol. 125, no. 2, pp. 155-164, 1995.

[9] A. Gabbuti, L. Romano, P. Blanc et al., "Long-term immunogenicity of hepatitis B vaccination in a cohort of Italian healthy adolescents," Vaccine, vol. 25, no. 16, pp. 3129-3132, 2007.

[10] X. Liu, Y. Takeuchi, and S. Iwami, "SVIR epidemic models with vaccination strategies," Journal of Theoretical Biology, vol. 253, no. 1, pp. 1-11, 2008.

[11] J. Li, Y. Yang, and Y. Zhou, "Global stability of an epidemic model with latent stage and vaccination," Nonlinear Analysis: Real World Applications, vol. 12, no. 4, pp. 2163-2173, 2011.

[12] C. Castillo-Chavez and Z. Feng, "To treat or not to treat: the case of tuberculosis," Journal of Mathematical Biology, vol. 35, no. 6, pp. 629-656, 1997.

[13] Z. Feng, C. Castillo-Chavez, and A. F. Capurro, "A model for tuberculosis with exogenous reinfection," Theoretical Population Biology, vol. 57, no. 3, pp. 235-247, 2000.

[14] Z. Feng, M. Iannelli, and F. Milner, "A two-strain tuberculosis model with age of infection," SIAM Journal on Applied Mathematics, vol. 62, no. 5, pp. 1634-1656, 2002.

[15] Y. L. Yang, J. Q. Li, Z. E. Ma, and L. J. Liu, “Global stability of two models with incomplete treatment for tuberculosis," Chaos, Solitons E Fractals, vol. 43, no. 1-12, pp. 79-85, 2010.

[16] O. Neyrolles, R. Hernandez-Pando, F. Pietri-Rouxel et al., "Is adipose tissue a place for Mycobacterium tuberculosis persistence?" PLoS One, vol. 1, no. 1, article e43, 2006.

[17] Science Daily, "Tuberculosis Bacillus Hides From Immune System In Host's Fat Cells," http://www .sciencedaily.com/releases/2006/12/061221074735.htm.

[18] S. M. Blower and C. Tom, "Modeling the emergence of the 'hot zones' tuberculosis and the amplification dynamics of drug resistance," Nature Medicine, vol. 10, pp. 1111-1116, 2004.

[19] X. Yang and L. Chen, "Permanence and positive periodic solution for the single-species nonautonomous delay diffusive models," Computers $\mathcal{E}$ Mathematics with Applications, vol. 32, no. 4, pp. 109116, 1996.

[20] P. van den Driessche and J. Watmough, "Reproduction numbers and sub-threshold endemic equilibria for compartmental models of disease transmission," Mathematical Biosciences, vol. 180, pp. 29-48, 2002.

[21] J. P. LaSalle, The Stability of Dynamical Systems, Regional Conference Series in Applied Mathematics, SIAM, Philadelphia, Pa, USA, 1976. 


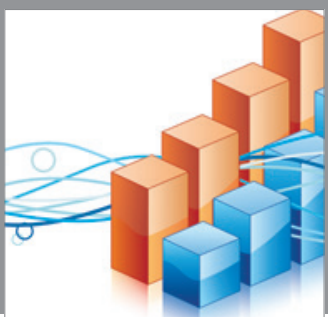

Advances in

Operations Research

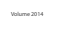

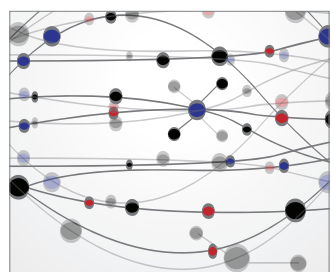

\section{The Scientific} World Journal
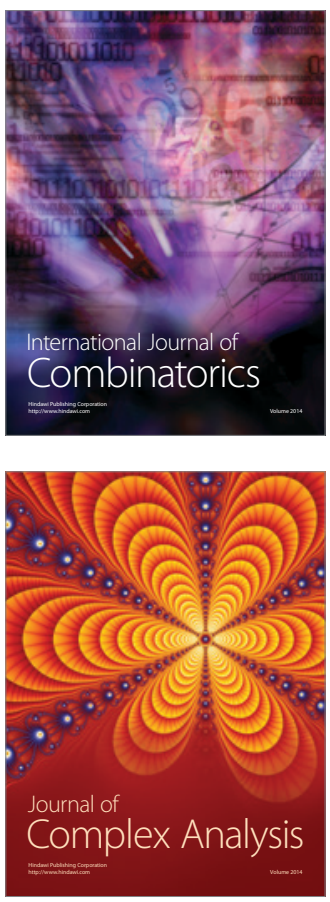

International Journal of

Mathematics and

Mathematical

Sciences
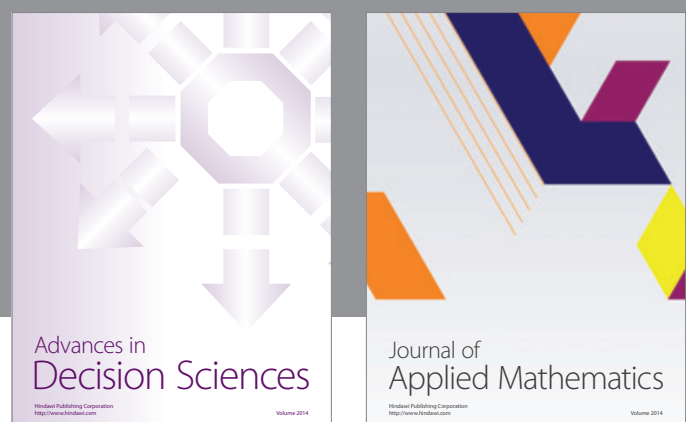

Journal of

Applied Mathematics
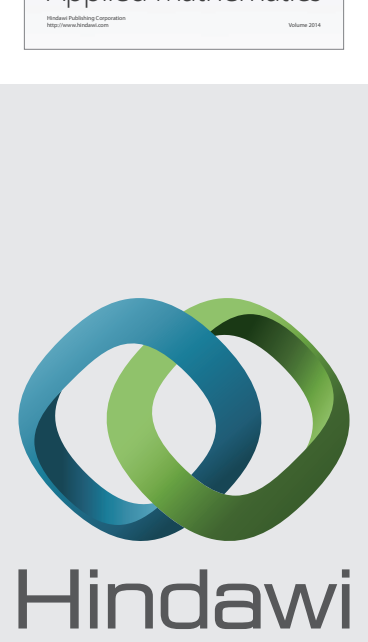

Submit your manuscripts at http://www.hindawi.com
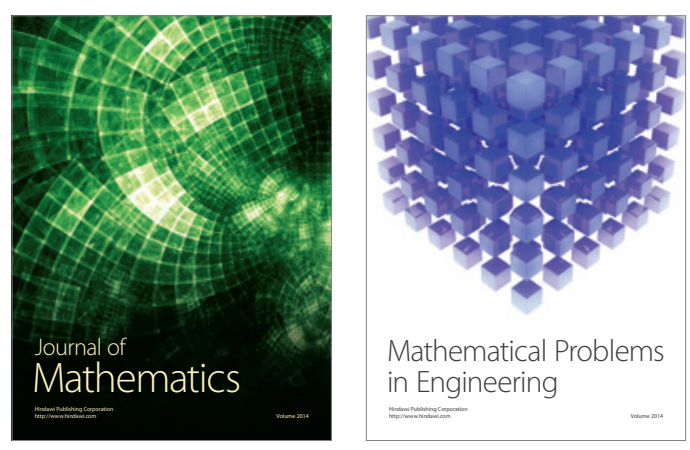

Mathematical Problems in Engineering
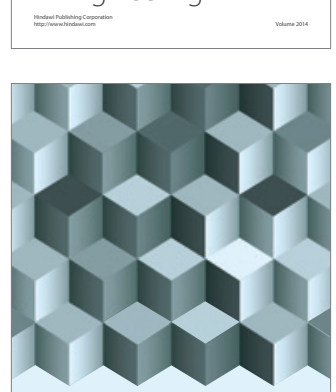

Journal of

Function Spaces
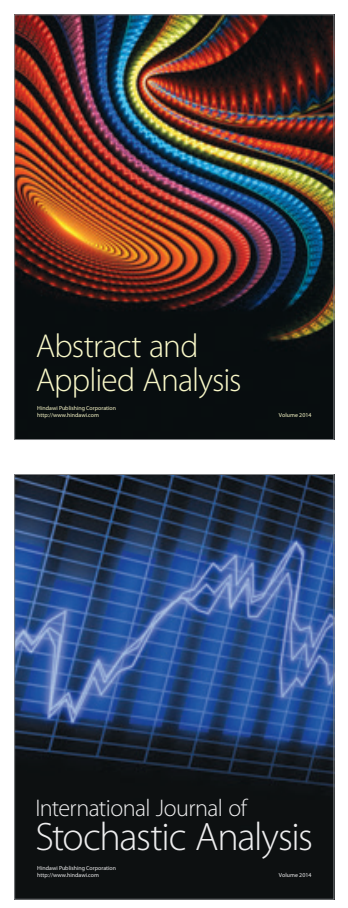

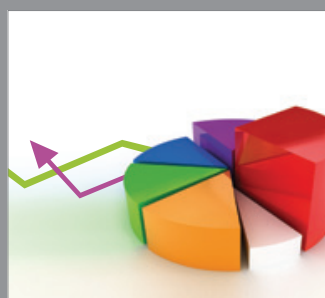

ournal of

Probability and Statistics

Promensencen
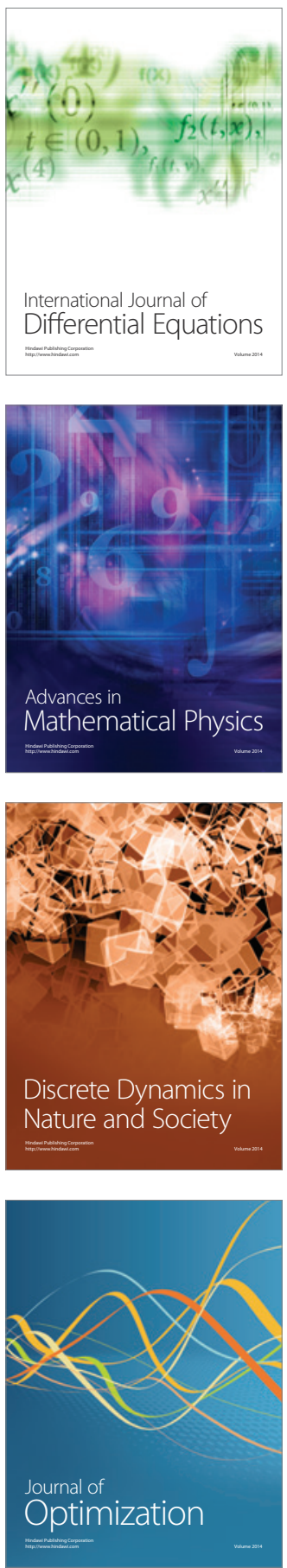